\title{
Excessive sweating: an uncommon sign of basilar artery occlusion
}

\author{
Adnan Awada, Ahmed Ammar, Saad Al-Rajeh, M Borollosi
}

\begin{abstract}
Excessive sweating of the face and, to a lesser degree, of the thorax and limbs, occurred after basilar artery occlusion in a 15 year old Saudi boy. Magnetic resonance imaging (MRI) showed a limited bilateral paramedian infarction of the rostral ventral pons. Basilar artery occlusion in children and adolescents is a rarity. Excessive sweating following such a stroke is rare in any age group. Clinico-anatomical correlations are discussed.
\end{abstract}

Signs and symptoms of basilar artery occlusion are described in many articles and textbooks, ${ }^{1-4}$ but none mention excessive sweating as a sign of this disorder. We observed a case of basilar artery occlusion where hyperhidrosis was one of the most striking manifestations. In addition, a magnetic resonance imaging (MRI) was performed which allowed relatively precise clinico-anatomical correlations.

\section{Case report}

A 15 year old Saudi boy complained of sudden unusual headache at $7 \mathrm{pm}$ on the 6 September 1988 while he was sitting. Some seconds later, he vomited and lost consciousness. He was admitted to another hospital where the diagnosis of subarachnoid haemorrhage was suspected. Cerebrospinal fluid (CSF) was normal and the patient was referred to our hospital the next morning. The parents did not notice any unusual symptoms before that evening. The patient was enjoying a normal life and did not perform any sport or excessive physical activity that day.

Twelve hours after the first symptoms he remained in a coma. Rectal temperature was $37 \cdot 1^{\circ}$. The neck was supple but flexion produced bilateral decerebrate rigidity. Pupils were $3 \mathrm{~mm}$, equal and reactive to light. No ocular deviation or nystagmus was noted. Respiration, although regular, was laboured due to excessive bronchial secretions. An endotracheal tube was inserted and the patient was put on a respirator for 24 hours.

Serum electrolytes measured at admission showed the following values: sodium $140 \mathrm{mmol} / \mathrm{l}$, potassium $3.8 \mathrm{mmol} / \mathrm{l}$, chloride $106 \mathrm{mmol} / 1$. Brain computerised tomography (CT) scan, performed immediately after admission, was normal. Transfemoral selective angiogram of the cerebral arteries showed an occlusion of the basilar artery at its upper third (fig 1). No obvious underlying arterial disease was seen. Electrocardiogram, echocardiogram, Holter monitoring, coagulation tests, lipid profile, syphilitic serology and haemoglobin electrophoresis were normal or negative.

The level of consciousness of the patient improved rapidly. Three days after admission, he was opening his eyes and could follow instructions. Nevertheless, severe tetraplegia and bilateral facial paresis was still present. The tongue and palate were paralysed. Face and body pain sensation and ocular movements were normal. A MRI carried out during the third week showed the limits of the brainstem infarction: it involved, on sagittal view, the rostral half of the ventral pons (fig $2 a)$ and, on axial views, the anterior part of the territories of the paramedian branches of the basilar artery at this level (fig 2b). The infarct was more extended posteriorly on the right side.

An unusual sweating disorder was noticed from the second hospital day. Excessive bilateral sweating of the face and, to a lesser degree, of the thorax and limbs was noted. This occurred independently of the temperature of the patient $\left(37^{\circ} \mathrm{C}\right)$ and the air-conditioned room $\left(23-25^{\circ} \mathrm{C}\right)$. Sweating was very obvious when the patient was angry, distressed or in pain and increased dramatically when hot food was administered through the nasogastric tube. This exessive sweating decreased slowly and disappeared after 30 days.

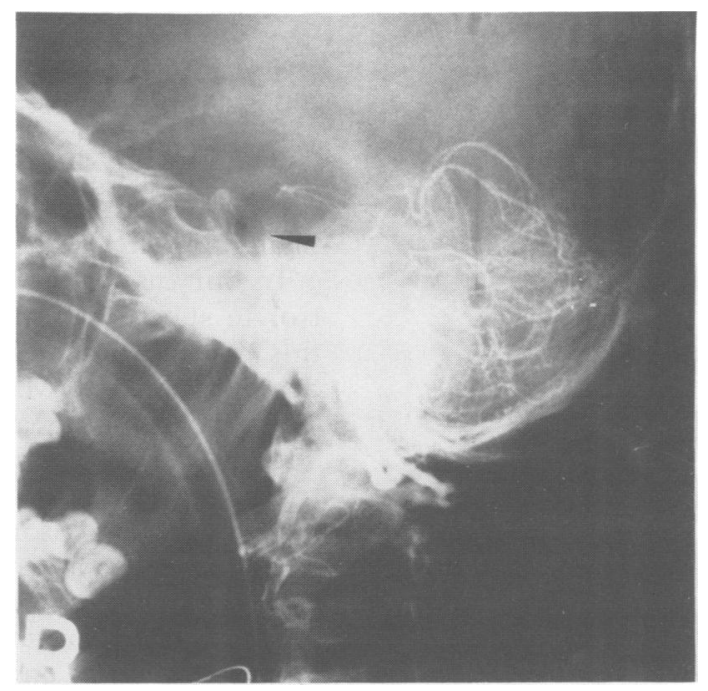

Figure 1 Selective right vertebral angiogram: occlusion of upper third of the basilar artery (arrowhead). 


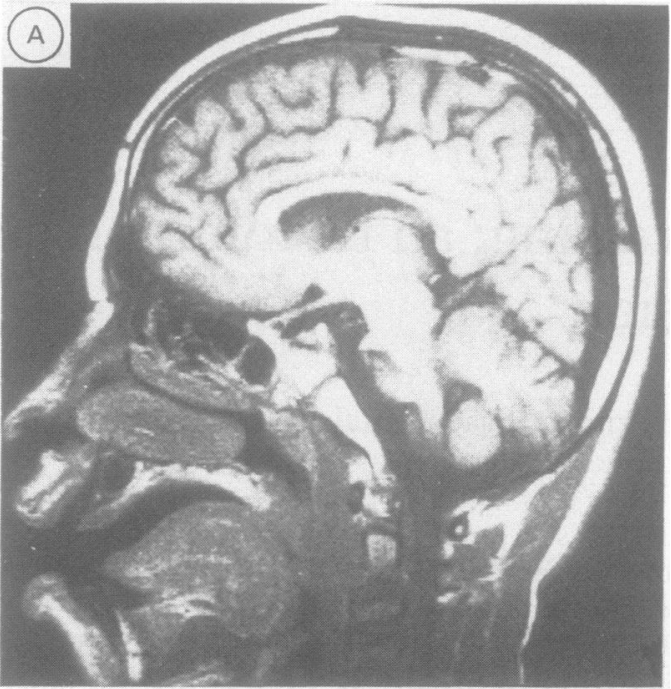

Figure 2 Post-gadolinium $T$, weighted MRI (a) sagittal view: hyperintense signal suggesting an infarction of the rostral ventral pons; (b) axial view: the hyperintense signal involves the ventral pons and extends posteriorly in the paramedian regions suggesting an infarction of the territories of both paramedian branches of the basilar artery.

Two months after onset, the patient was still severely tetraplegic with brisk reflexes and bilateral Babinski sign. Upper limbs were fixed in flexion and lower limbs in extension. Sensation was normal. Bilaterial facial paresis predominantly of the lower part of the face was still present and he exhibited intermittent spastic laughing. He required feeding by nasogastric tube due to the paralysis of the tongue, palate and pharynx. Sweat function remained normal.

\section{Discussion}

Excessive sweating has been reported in many lesions of the central nervous system. It is a classic feature of tetraplegia and high level paraplegia after spinal cord trauma and is usually associated with episodes of autonomic hyperreflexia. ${ }^{5}$ It has also been described in hemispheric strokes, ${ }^{5-6}$ mainly when the cerebral lesion involved the opercular cortex. In these cases, hyperhidrosis was limited to the contralateral side of the body. Excessive sweating, however, is very rare in stroke. It was recorded only in three of 633 consecutive strokes by Lubar et al. ${ }^{6}$ We found only one reported case of excessive sweating associated with brainstem infarction. ${ }^{7}$ Hyperhidrosis was not mentioned in the clinical discussion and necropsy examination showed an extensive bilateral infarction of the basis pontis.

In our case, the temperature and the electrolyte status were normal on admission and this suggested that the brainstem infarction demonstrated by MRI was the cause of the excessive sweating and not its consequence. The infarction was limited and involved restrictively the corticospinal tracts at the upper pontine level. Sensory and oculomotor pathways as well as sympathetic fibres were preserved. In their recent article, Labar et $a l^{6}$ postulated the presence of a pathway which inhibits sweating on the contralateral side of the body. This pathway originates in the cortex, possibly of the operculum, making

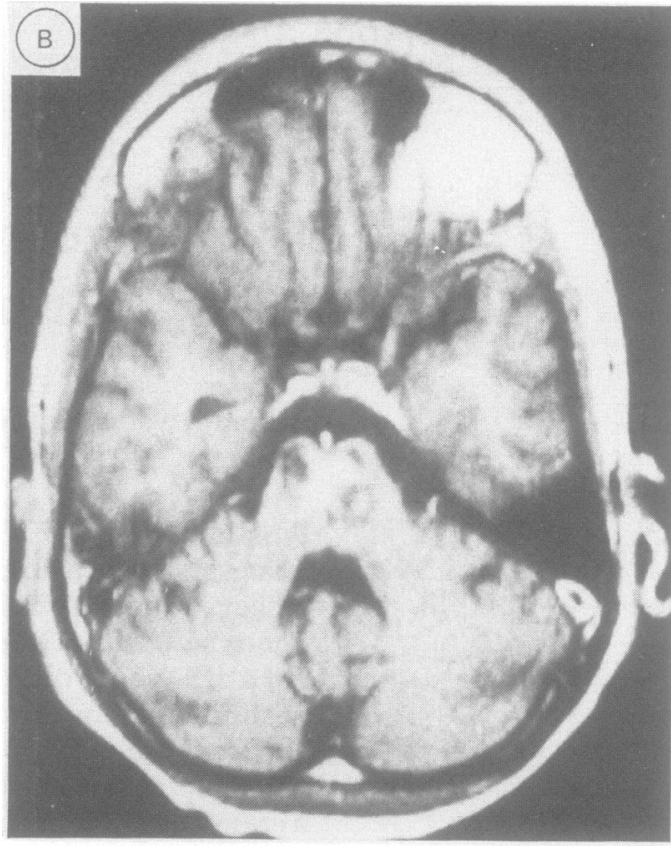

terminal connections with the contralateral thoracic spinal cord. Our observations, as well as those of Fisher, ${ }^{7}$ suggest that at the level of the pons, the fibres of this putative pathway would be mixed with or very close to the corticospinal tract.

Excessive sweating in our case did not last more than four weeks, longer than the one to three days reported in four cases of hemispheric stroke by Labar et $a l,{ }^{6}$ but much less than the one or more year reported in spinal cord lesions. ${ }^{8}$ Our inference is that the duration of hyperhidrosis may be related to the site of the nervous lesions and compensatory mechanisms are much stronger in higher lesions.

Finally, our case was also uncommon because of the age of the patient. Until recently, only 30 cases of basilar artery occlusion before the age of 16 years have been reported. ${ }^{9}$ This disorder represents around $15 \%$ of cerebral artery occlusions in older patients. ${ }^{10}$ There is no clear explanation for this rarity. It may be due to the fact that the main cause of basilar artery occlusion is atherosclerosis, ${ }^{3}$ a very uncommon disease in children and adolescents.

We are grateful to Professor D Maclean, Consultant Neurologist at the King Fahd Hospital-National Guard, Riyadh, for
reviewing the manuscript.

1 Kurbick CS, Adams RD. Occlusion of the basilar artery. A clinical and pathological study. Brain 1946;69:73-121.

2 Cravioto H, Rey Bellet J, Prose PH, Feigin I. Occlusion of the basilar artery. A clinical and pathological study of 14 the basilar artery. A clinical and pathological

3 Castaigne P, Lhermitte F, Fautier JC, et al. Arterial occlusions of the vertebrobasilar system. A study of 44 patients with postmortem data. Brain 1973;96:133-54.

4 Adams RD, Victor M. Cerebrovascular diseases. In: Principles of Neurology, Fourth ed. New York: McGraw Hill,

5. Appenzeller $O$. The autonomic nervous system. Amsterdam: Elsevier, 1982

6 Labar DR, Mohr JP, Nichols FT, Tatemichi TK. Unilateral hyperhidrosis after cerebral infarction. Neurology 1988; 38:1679-82.

7 Fisher CM. Bilateral occlusion of basilar artery branches. $J$ Neurol Neurosurg Psychiatry 1977;40:1182-9.

8 Kneisley LW. Hyperhidrosis in paraplegia. Arch Neurol 1977;34:536-9.

9 Mares R, Pon A, Nolla J, Mirosa F, Munoz JA. Occlusion of the basilar artery in a 7-year old boy. $J$ Neurol Neurosurg Psychiatry 1987;50:494-5.

10 Silverstein A. Acute infarctions of the brainstem in the distribution of the basilar artery. Confin Neurol 1964;27: 37-61. 